Instructions for authors, subscriptions and further details:

http://ijelm.hipatiapress.com

\title{
List of Reviewers
}

Date of publication: January $16^{\text {th }}, 2019$

Edition period: January 2019-July 2019

To cite this article: IJELM Editors. (2019). List of Reviewers. International Journal of Educational Leadership and Management, 7(1), 138. Doi: 10.17583/ijelm.2019.4008

To link this article: http://dx.doi.org/10.17583/ijelm.2019.4008

\section{PLEASE SCROLL DOWN FOR ARTICLE}

The terms and conditions of use are related to the Open Journal System and

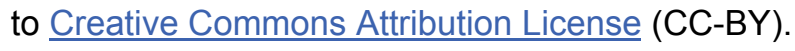




\section{List of Reviewers}

Thank you to 2018 reviewers. On behalf of the International Journal of Educational Leadership and Management we deeply appreciate reviewers contributions to the quality of this journal. The journal owes this debt with those who have been peer reviewers during this period. Yours sincerely,

Gisela Redondo-Sama \& Marta Camarero-Figuerola (Editors)

Miriam Agreda Montoro, Universidad de Jaén

Adriana Aubert, Universidad de Barcelona

Andréa Castaño Sánchez, Universidad Nacional de Educación

Miguel Diaz Delgado, Instituto de Investigaciones Sobre la Universidad y la Educación, UNAM

Inmaculada García-Martínez, Universidad de Granada

Nina Hidalgo Farran, Universidad Autónoma de Madrid

María Lina Higueras Rodríguez, Universidad de Granada

Saadet Kuru Cetín

Margarita Martín Martín, Universidad Complutense de Madrid

Ana Martín Romera, Universidad de Granada

Cynthia Martínez-Garrido, Universidad Autónoma de Madrid

Estefanía Martínez Valdivia, Universidad de Jaén

Irene Moreno Medina, Universidad Autónoma de Madrid

Maria Ramis Salas, Universidad de Barcelona

Guillermo Rodríguez Molina, Universitat Autónoma de Barcelona

Ignacio Santa Cruz, Universidad Autónoma de Barcelona

Juana-María Tierno-García, Universitat Rovira i Virgili 